\title{
Influence of Three Different Scanning Techniques in Full-Arch Implants Digital Impression Using Intraoral Scanners: A Randomized Controlled Cross-Over Trial
}

\author{
Adolfo DI FIORE ${ }^{1}$, Roberto MENEGHELLO ${ }^{2}$, Gianpaolo SAVIO ${ }^{3}$, Lorenzo \\ GRAIFF $^{1}$, Carlo MONACO ${ }^{4}$, Edoardo STELLINI ${ }^{5}$
}

\begin{abstract}
Aims: The purpose was to compare three different techniques of scanning in a full-arch digital impression. Digital impression accuracy and time of scanning of inexperienced operators were assessed.

Methods: A polymethyl methacrylate acrylic (PMMA) model of an edentulous mandible with six scan-abutment was used as a master model and its dimensions measured with a coordinate measuring machine. Three different techniques of scanning (MetA; MetB and MetC) were applied on the master model with an intraoral scanner (Zfx, Zimmer-Biomet). Nine students were divided in three groups. All students were instructed how to use the technique assigned. Each group knows only the scanning technique assigned. Each student performed 3 scans. The acquisition times (minutes) of each scan were recorded. All the digital impressions were imported and analysed with a CAD software (Rhinoceros 5.0, RobertMcNeel) and compared with the master model, obtaining the scanning accuracy. One-way analysis of variance with a post hoc analysis (Bonferroni's test) was used to compare the three groups.

Results: Statistically significant difference was present between MetA, MetB and MetC $(\mathrm{p}$ value $=0.02)$. The mean total treatment time was of $14.5 \mathrm{~min}$ (SD $4.5 \mathrm{~min}$ ) for the group MetA; $11.5 \mathrm{~min}$ for MetB and 12.1 min for MetC.

Conclusions: The scanning methods have a great influence on the accuracy of full-arch implants. The time analysis showed that with the increasing of the

\footnotetext{
1 Adjunct Professor, Department of Neuroscience, Dental School, University of Padova, Italy.

2 Assistant Professor, Departments of Management and Engineering, University of Padova, Italy.

3 Assistant Professor, Departments of Civil, Environmental and Architectural Engineering,

University of Padova, Italy.

4 Researcher, Assistant Professor, Division of Prosthodontics and Maxillofacial Rehabilitation, Department of Biomedical and Neuromotor Sciences (DIBINEM), Alma Mater StudiorumUniversity of Bologna, Bologna, Italy.

5 Dean, Dental School, University of Padova, Italy.

DOI: 10.17932/EJOH.2020.022/ejoh_v01i1003
} 
Influence of Three Different Scanning Techniques in Full-Arch Implants Digital Impression Using

Intraoral Scanners: a Randomized Controlled Cross-Over Trial

number of scans the scan time decreases.

Keywords: digital impression, scanning techniques, intraoral scanner, accuracy, strategy

\section{Introduction}

The accuracy is a primary factor for long term clinical success and survival of an implant-supported fixed dental prosthesis (FDP). However, the insufficient accuracy during the impression-making technique and/or manual steps during prosthesis fabrication may lead to misfit of the prosthesis and subsequent to technical, mechanical, and biological complication such as occlusal discrepancies screw or abutment loosening, fracture of the prosthetic components, implant fractures, and loss of osseointegration (1-5). Today, conventional impression with different techniques and materials represent a commonly used procedure in general dental practice (6). With the implementation of the intraoral scanner (IOS), many prosthetic procedures as tray selection, dispensing and setting of impression materials, splinting the impression coping pick-up have been eliminated, while patient comfort is increased, the protocols have been simplified, less potential gag reflex, no potential deformation of the impression and time to clinical treatments have been reduced $(7,8)$. However, there are different obstacles and deficiencies to address in intraoral impressions. The additional cost of purchasing an IOS, the learning curve for adjusting to the new methodology, scanner displacement during the scanning accuracy are the major limitations at the digital workflow. An important factor in clinical handling is the scanning strategy. In the dental market there are different IOS, which differ for the scanning technologies (9). Thus, there is a low knowledge on the relationship between accuracy, repeatability and methods of scanning. In the different field of medicine, several researches have conducted to know the learning curve of new devices $(10,11)$. In literature the majority of studies on intraoral digital impression investigated the accuracy of digital impressions (12-15) or compared the working time of digital and conventional impression methods (16).

Only two studies have been conducted to evaluate the methods of scanning $(17,18)$ and one article evaluated the experience curve with two different IOS (19). The purpose of this randomized crossover in vitro study was to compare three different techniques of scanning in a full-arch digital impression and the time of scanning of digital impression by inexperienced operators. 


\section{Materials and Methods}

Master Model

A virtual model of a mandibular edentulous with six scan-abutment positioned vertically at different height was designed by means of a computer-aided design (CAD) software (SolidWorks Corporation). The shape of the master model resembled a mandibular implant-supported full arch rehabilitation. Six scanabutments were positioned symmetrically corresponding to the mandibular first molars, first premolars, and lateral incisors. All the scan-abutment geometries (i.e. regular cylinders) were parallel to each other with a diameter of $4 \mathrm{~mm}$ and incorporated into the master model. The regular geometry of scan-abutments was chosen, following metrologists expertise due to: i) the favourable design to perform the calibration measurements using a coordinate measuring machine, ii) the unfavourable design to stress stitching algorithm/procedure adopted by scanning systems. Subsequently the master model, with integrated scan-abutments, was manufactured in PMMA by a CNC machine tool to serve as a clinically relevant simulation model (master model). PMMA as the master model material was adopted in order to ensure adequate stiffness, strength, dimensional stability and to eliminate the need for spraying the model. Soft tissue was simulated using silicone (Vestogum, $3 \mathrm{M}$ ) in order to enable accurate measurements (Fig.1).

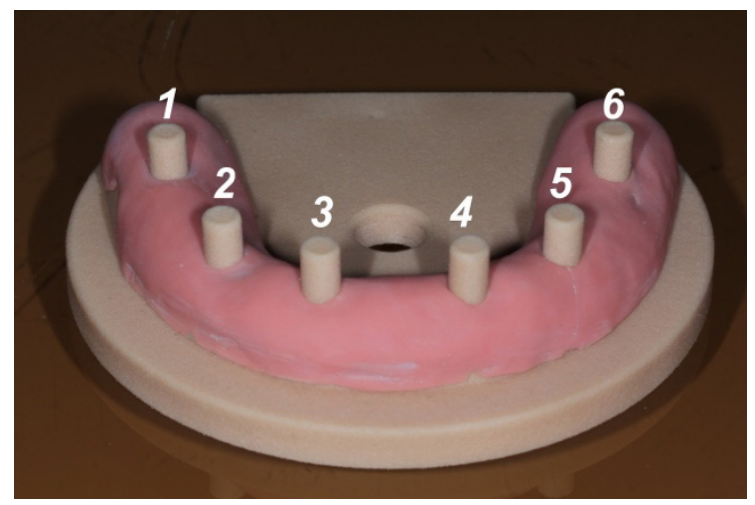

Figure 1. Master Model

Calibration Plan and Procedure

The experimental campaign consisted in three phases: 1) calibration of the master model using the OGP SmartScope Flash CNC 300 with the contact system; 2) acquisition of the master model by expert operators with eight Ios; 3) recurrent calibration of the master model using the CMM with the contact system. The master model was measured with a coordinate measuring machine (CMM) (OGP SmartScope Flash,CNC 300), an optomechanical system that is capable 
of moving a measuring probe to determine the spatial coordinates of points on a workpiece surface. The measuring system is capable of a $3 \mathrm{D}$ maximum error assessed as $\mathrm{E} 3$-xyz $(\mathrm{L})=2.8+5 \mathrm{~L} / 1000 \mu \mathrm{m}$ (with $\mathrm{L}$, in millimeters, equal to the measured distance, according to ISO 10360 standard). A high-accuracy contactprobe with ruby sphere of $1.5 \mathrm{~mm}$ of diameter was used to measure the points of the scan-abutment upper and lateral surfaces to locate them in a $\mathrm{x}, \mathrm{y}$ and $\mathrm{z}$ coordinate reference frame. Recurrent verification of the master model was required between scanning sessions with Ios to check the dimensional stability of the master model. The calibrations of the master model were performed based on the points in Figures 2a and 2b: a partial, preliminary, reference frame on the master model was defined, then the scan-abutments were measured. For each scan-abutment a plane (Fig 2c) and a cylinder (Fig. 2d) were identified, adopting specific sets of points. Finally, the position of each scan-abutment was computed as the intersection between the plane and the axis of the cylinder. The coordinates of the probed points and intersections were transferred into a $3 \mathrm{D}$ CAD geometric modelling software program (Rhinoceros 5.0) and analysed with a specific evaluation protocol, developed in IronPython, to estimate the position and orientation of each scan-abutment. This procedure was repeated five times. A mean of the five measurements performed with the CMM was used as reference position of scan-abutments for the evaluation of the accuracy of each digital impression obtained by eight different IOS.

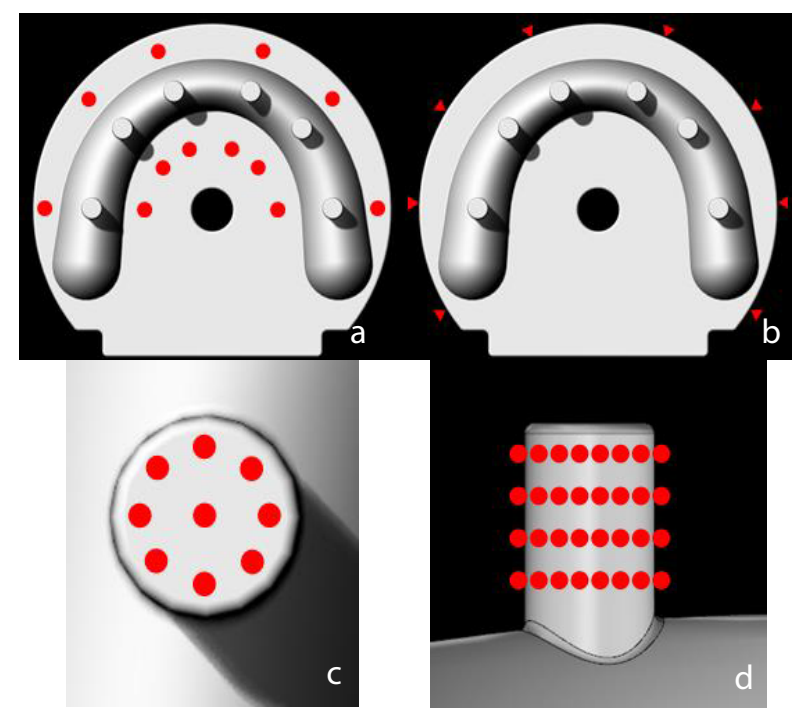

Figure 2. The measurements of the master cast: a) Points in the XY plane. b) Points on the outer circumference. c) Measurement of 9 points on the upper plane of the scan-abutment. d) Acquisition of 4 circular sections (260 points) perpendicular to the axis of the scan-abutment. 


\section{Study Design and Impression Procedure}

The study was designed as a blind randomized controlled cross-over experiment students of dental school at second year, without any previous digital implant impression procedure experience. The sample was formed by 3 students divided to three groups of scanning's techniques. The impression was made in a controlled-temperature environment $\left(22^{\circ} \mathrm{C} \pm 2^{\circ} \mathrm{C}\right)$ with a relative humidity of $45 \% \pm 10 \%$. Through a randomization computer-generated were assigned the three different techniques of scanning each group. Three different techniques of scanning (classified as MetA; MetB and MetC) (Fig. 3) performed by an intraoral scanner (Zfx, Zimmer-Biomet) were described by one expert operator (A.D.F.) to groups. All operators were instructed how to use the device, the technique assigned and follow the definite protocol. Each group knows only the scanning technique assigned. Each student performed 3 scans. For each methodology 9 detections were performed. The acquisition times (minutes) of each scan were recorded. The digital impression from each group were executed on three separate days. The operators in each group performed the acquisition alternately. The scanning technique MetA consisted of positioning the intraoral scanner parallel to the occlusal surface of the scan-abutments in position 46. Move the scanner in parallel to the occlusal surface toward the mesial surface, positioning the intraoral scanner parallel to the facial surface of the scan-abutments in position 46 and move scanner in parallel to the facial surface toward the mesial surface, positioning the intraoral scanner parallel to the lingual surface of the scan-abutments in position 46 and move scanner in parallel to the lingual surface toward the mesial surface. Continue the acquisition of scan-abutment to the element in position 36 (Fig. $3 a)$. The scanning technique MetB consisted of positioning the intraoral scanner parallel to the occlusal surface of the scan-abutments in position 46. Move the scanner in a rotating motion from occlusal to lingual and forth until arriving the scan abutments in position 36 . To enable interdental scanning, the scanner has to be held slightly shifted and rotated (Fig. 3b). The technique of scanning MetB was recommended by the manufacturer. The scanning technique MetC consisted of positioning the intraoral scanner perpendicular to the midline facing the facial surface of the scan-abutment in position 42 . Continue scanning in an occlusal direction and then in the distal direction from the scan-abutment 42 to acquire the scan-abutment in position 44 and in position 46 . The lingual surfaces were scanned through the execution of a semicircle movement during the scan of the occlusal surface. Once concluded the scanning of the 4th quadrant, it starts to scan the facial surface of the scan-abutment in position 32 in the same way as the 4th quadrant (Fig. 3c). The steps of acquisition of the master model were divided into three phases: 1) calibration of the master model using the OGP SmartScope Flash CNC 300 with contact system; 2) acquisition of the master model by students 
divided into three groups with intraoral scanner; 3) re-calibration of the master model using the OGP SmartScope Flash CNC 300 with contact system.
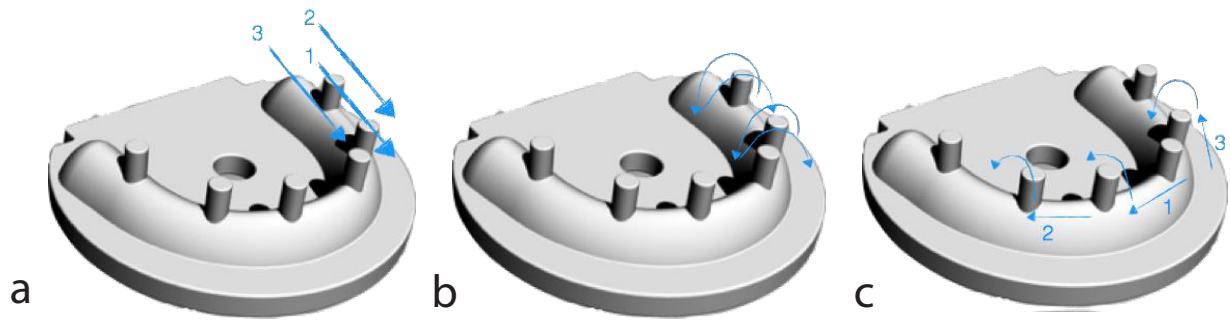

Figure 3. Scanning technique: a) MetA; b) MetB; c) MetC.

\section{Accuracy Assessment}

The STL file imported in the 3D CAD software (Rhinoceros 5.0) was furtherly processed to perform 3D position and distance analysis. A software plug-in called "Scan-abut" was developed in order to automatically segment the mesh of the scan-abutment by curvature analysis (Fig.4). The segmented dataset was then filtered (reduced), with $2 \sigma$ Gaussian criterion, and two independent fitting were computed to calculate the upper plane surface and the later cylindrical surface of the scan-abutment (Fig.5a; Fig. 5b). From the intersection of the cylinder axis with the plane, a centre point was assessed, which identifies the scan-abutment position (Fig. 5c).
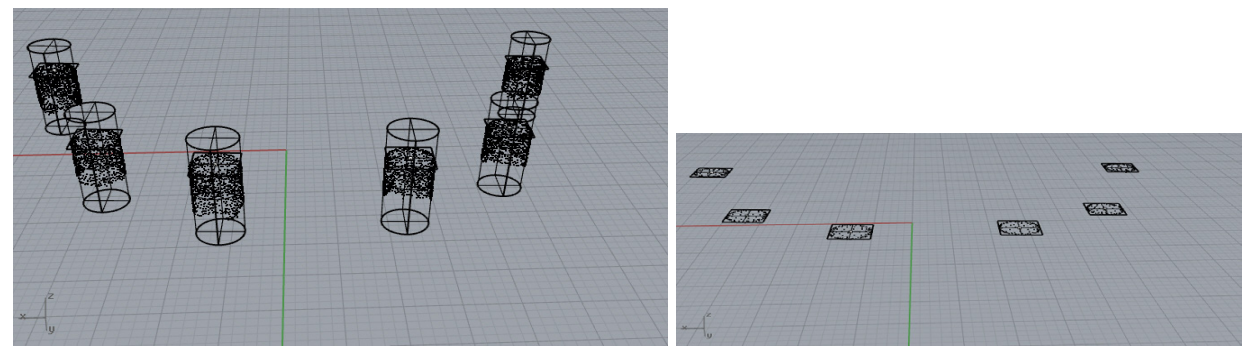

Figure 4. The software application called "Scan-abut" was realized as a plug-in for Rhinoceros. The software "scan-abut" segments automatically the surfaces of the scan-abutment (cylindrical area and plan area). 

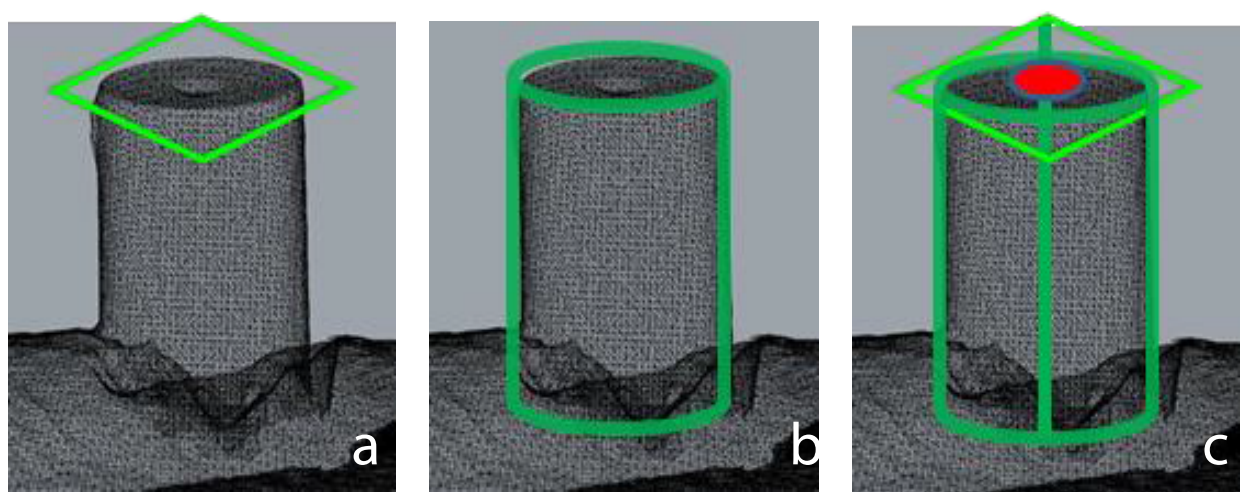

Figure 5. Construction of the geometric elements during calibration master model: a) construction of the plan of fitting through 9 points measured above. b) Construction of the cylinders of fitting on 4 circular sections. c) Intersection of the axis of this cylinder with the upper floor to define a reference point for each individual scan-abutments.

To evaluate the absolute position error of scan-abutments, the six scan-abutment positions were aligned with the six reference positions measured by CMM, using a least-square best fitting algorithm. To investigate the accuracy of scanning systems with respect to arch length, a 3D intra-abutment distance was calculated as the 3D linear distance between paired scan-abutments (i.e. distance from scanabutment 1 to scan-abutment 6). A total of fifteen 3D distances, considering any combinations of six scan-abutments, were calculated for each digital impression (Fig. 6).

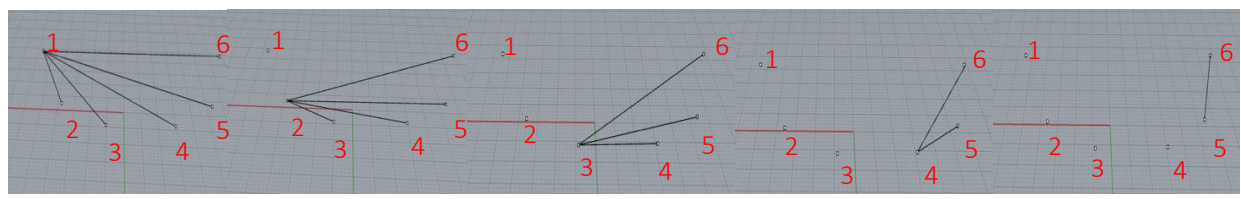

Figure 6. 3D Distance Analysis

The 3D distance error was calculated as the difference between the effective 3D distance between scan-abutments of the digital impression and the reference 3D distance between scan-abutments of the master model, measured with $\mathrm{CMM}^{15}$. The distance error was used to evaluate the relationship between the error and the distance as an indicator of the maximum permissible error (MPE) of the scanning system in accordance to ISO 10360 standards. Descriptive statistical analysis was performed for variables. The distance error was considered as the statistical unit. The accuracy of an impression was determined by two factors: trueness and precision (ISO 5725-1). The trueness described the deviation of the tested impression method from the original geometry, instead the precision 
described the deviations between the impressions. The mean of the distance error was considered the trueness, instead the standard deviation the precision. The primary variable was the distance $(\mu \mathrm{m})$ and the secondary variable was the time (minutes) employed in the execution of digital impression. One-way analysis of variance (ANOVA Test) with a post hoc analysis using Bonferroni's test was used to compare the three groups. The level of statistical significance was set as $\alpha=$ 0.05 and with a statistical power of $80 \%$.

\section{Results}

Twenty-seven digital impressions were performed with three different scanning methods. The total mean distance error for group with methods MetA was 0.26 $\mathrm{mm}$ (SD 0.21), for group with methods MetB was $0.085 \mathrm{~mm}$ (SD 0.075) and for group with methods MetC was $0.20 \mathrm{~mm}$ (SD 0.26). Statistically significant difference was present between MetA, MetB and MetC ( $\mathrm{p}$-value $=0.02)$. The post hoc analysis showed a no statistically significant difference between MetA and Met $C(p$-value $=0.53)$, instead statistically significant difference was present between MetA and MetB ( $p$-value=0.002), MetB and MetC $(p=0.04)$. The distance error analysis with regression line for the three different scanning techniques were reported in the graphics (Figure 7, 8,9). The graphics 2 showed that the error with the scanning method MetB had a good linear relation with the scan-abutment distance, instead the MetA e MetC presented no linear relation between error and distance, then more errors dispersion. The mean total treatment time was of $14.5 \mathrm{~min}$ (SD 4.5 $\mathrm{min}$ ) for the group MetA; $11.5 \mathrm{~min}$ (SD $3.8 \mathrm{~min}$ ) for the group MetB and $12.1 \mathrm{~min}(\mathrm{SD} 2.5 \mathrm{~min}$ ) for the group MetC. In the first section of scans with the technique of the group MetA the mean total treatment time was $18.6 \mathrm{~min}$, in the second section $15.3 \mathrm{~min}$ and in the third section 9.6 min. In the first section of scans with the technique of the group MetB the mean total treatment time was $15.3 \mathrm{~min}$, in the second section $11.6 \mathrm{~min}$ and in the third section 7.6 min with. In the first section of scans with the technique of the group MetC the mean total treatment time was $14.3 \mathrm{~min}$, in the second section $12.6 \mathrm{~min}$ and in the third section $9.3 \mathrm{~min}$. 
Adolfo DI FIORE, Roberto MENEGHELLO, Gianpaolo SAVIO, Lorenzo GRAIFF, Carlo MONACO, Edoardo STELLINI

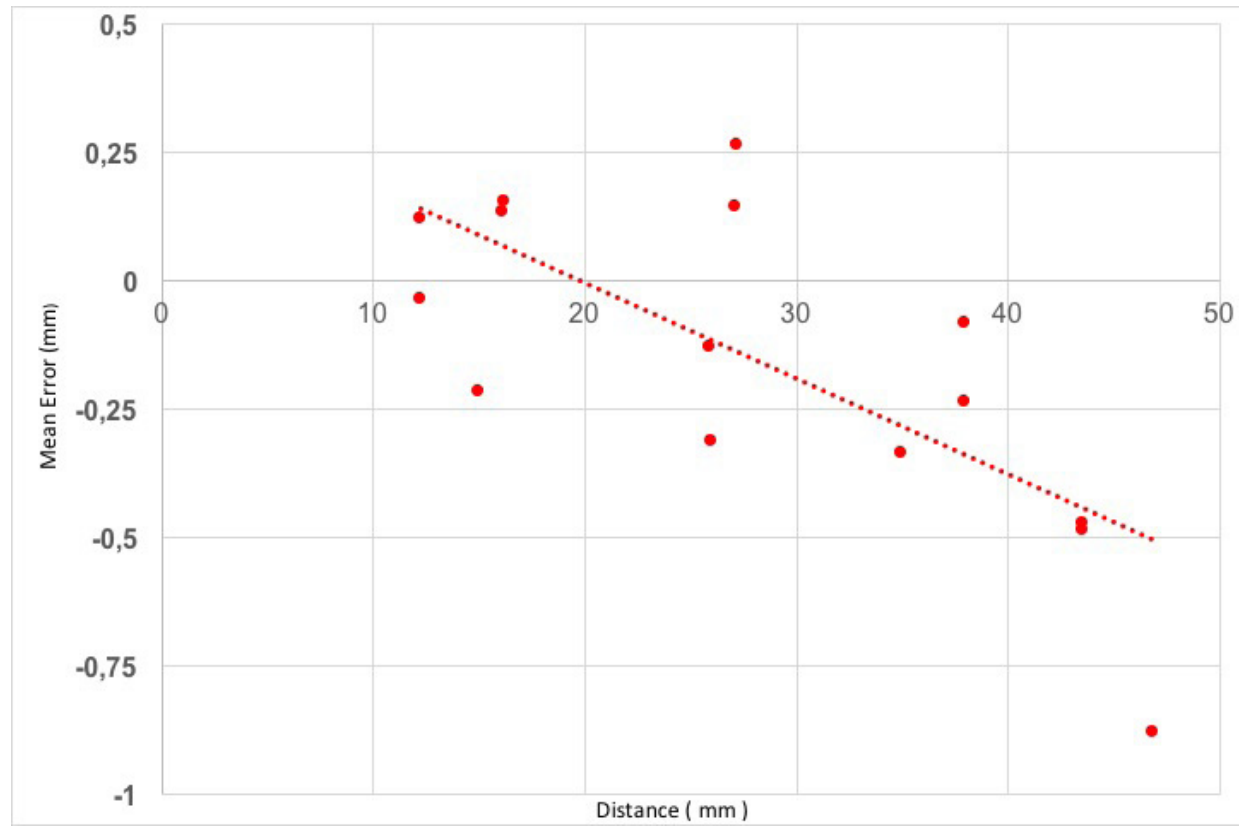

Figure 7. 3D distance analysis with regression line for MetA.

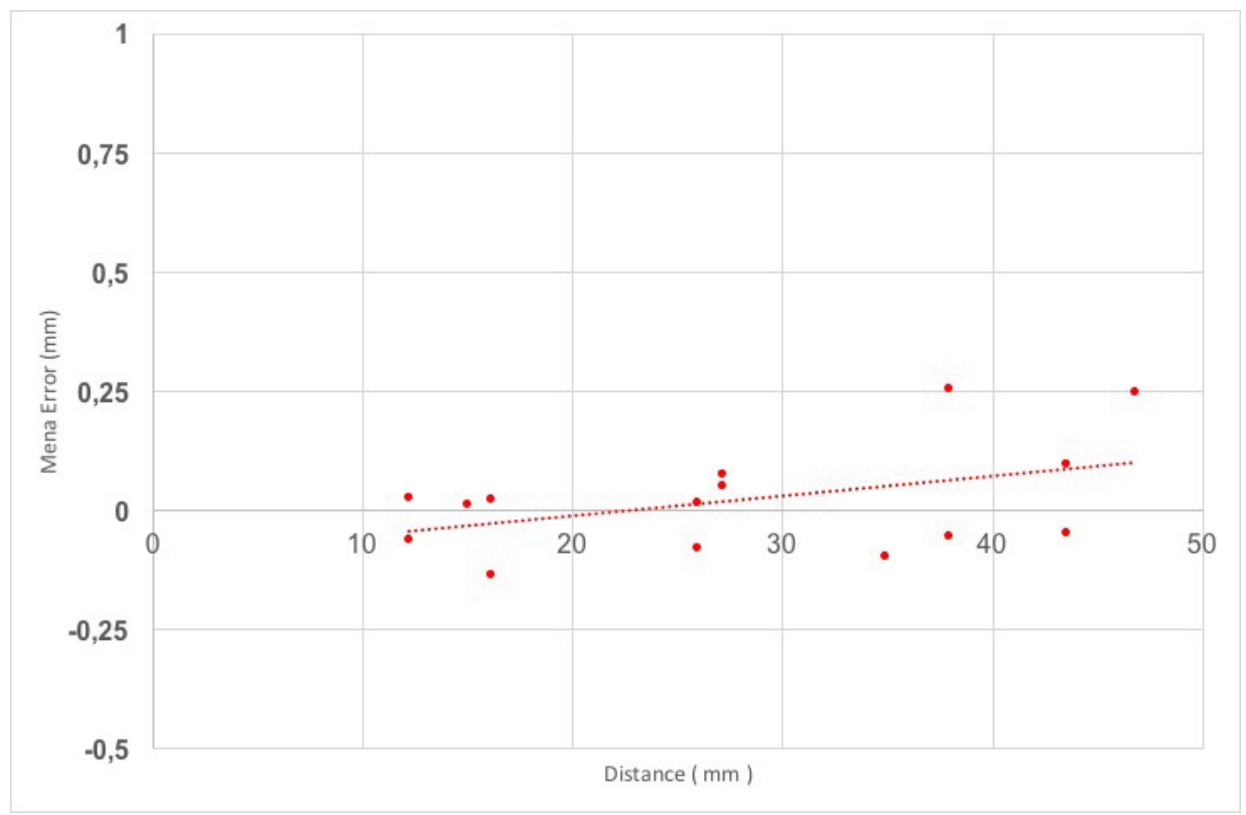

Figure 8. 3D distance analysis with regression line for MetB. 
Influence of Three Different Scanning Techniques in Full-Arch Implants Digital Impression Using Intraoral Scanners: a Randomized Controlled Cross-Over Trial

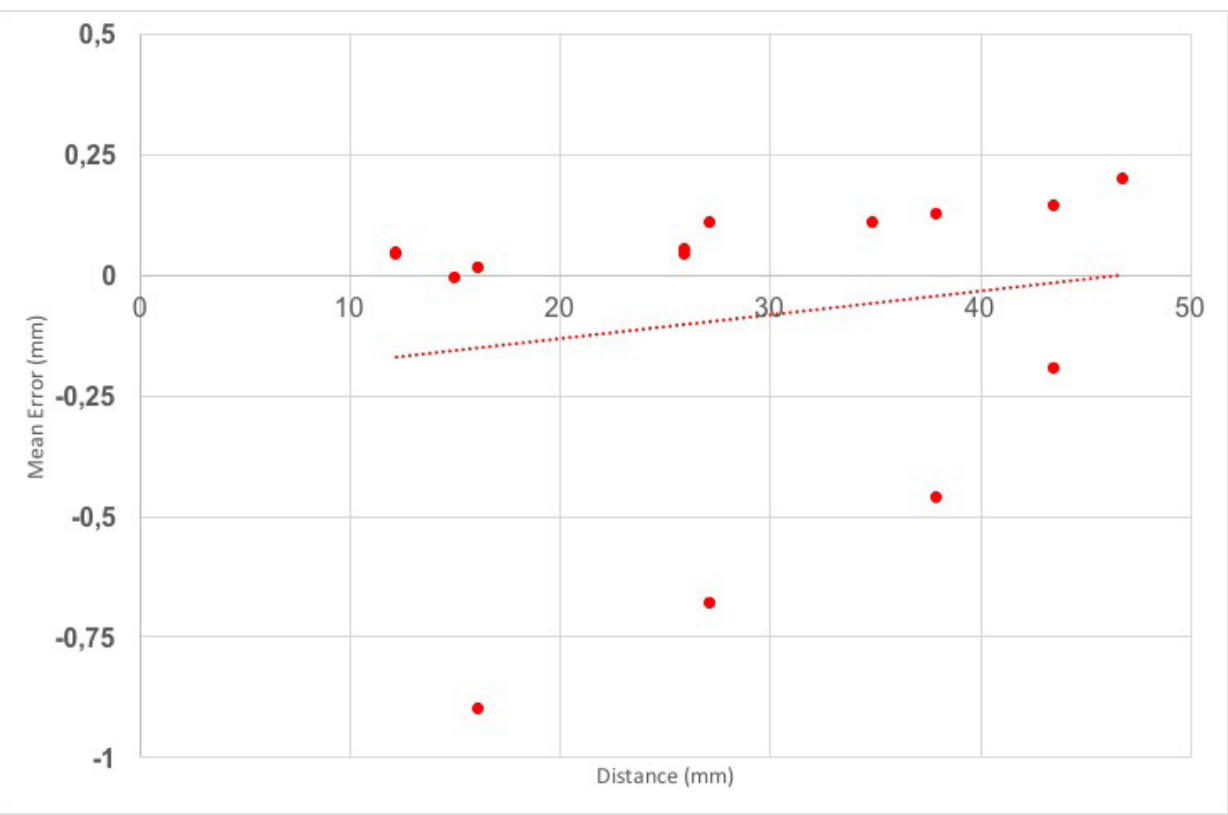

Figure 9. 3D distance analysis with regression line for MetC.

\section{Discussion}

To the best knowledge of the authors, this is one of the three studies that analysed the influence of three different techniques of scanning. Moreover, in this study the authors evaluated the learning curve of digital methodology by inexperienced operators. Based on the results, the scanning technique MetB presented a good linear relation with scan-abutment distance and the less mean distance errors. Thus, the scanning method MetB recommended by the manufacturer provided the highest accuracy in full-arch scans. The time analysis showed that with the increasing of the number of scans the scan time decreases. In literature just two articles compared the scans techniques for digital impression $(17,18)$. Ender and Mehl (17) used three different IOS: Lava COS (3M ESPE;Seefeld,Germany), Cerec Blucam (Sirona Dental System,Bensheim,Germany) and iTero (Caredet Ltd,Yehuda,Israel) with different scanning techniques. The first scanning protocols used with the Lava COS was recommended by the manufacturer and the second was taken from the publication by van der Meer et al. (9). The scanning methods used with the Cerec Blucam were developed by the same authors, instead the iTero scanning protocol was implemented within the acquisition software. However, only with the IOS Lava COS the authors used two scanning methods of which one recommended by the manufacturer. The accuracy of the digital impression obtained with the scanning methods recommended by the manufacturer was 
$45.8 \mu \mathrm{m}$, instead with another scanning method was $90.2 \mu \mathrm{m}$. Thus, the authors concluded that the scanning protocols have a large influence on the accuracy of digital impression. Müller et al (18) used one IOS Trios (3Shape, Copenaghen, Danimark) with three different scanning strategies. The scanning method B was recommended by the manufacturer. The authors concluded that the strategy B had the higher precision that the other methods, but no statistically significant difference between the scan techniques. Thus, the authors recommended the strategy B because provided the highest trueness and precision in full arch scans. In both researches, the master model representing a dentate patient (maxillary dentate jaw) made from a cobalt-chromium alloy, instead in our research used a model representing a mandibular edentulous with six scan-abutment positioned vertically at different height. However, the main difference was the methodology of evaluation of the accuracy. Ender et al. (17) and Müller et al. (18) scanned the model with an industrial reference scanner and the digital models of the reference scan, and the three scan methods were superimposed in software according to the best-fit algorithm. Instead, in our research a software called "Scan-abut" was realized as a plug-in for Rhinoceros 5.0 and the master model was measured with a coordinate measuring machine (CMM). This new methodology created by the authors is more objective and is not influenced by possible error due to the use of a reference scanner and a generic program for superimposed the STL file. Moreover, Ender et al. (17) and Müller et al. (18) used the position analysis to calculate the accuracy. Our research the 3D distance error was performed, because the graphics with regression line showed the relationship between error and distance. Errors dispersion might be related to incorrect software stitching process during the acquisition, then the scanning methods affect the accuracy of digital impression. In this research also the scanning time was examined. We recorded the trend of the scanning time with the three different techniques of scanning and after each session of scans the acquisition time of the master model decreases. From the first to the last session of scans there was a mean decrease of about $50 \%$ of the scanner. These results were comparable to another research (19). Kim et al. (19) compared the experience curves of two intraoral scanners and evaluated whether repeated scanning experience could change the scan time. With iTero (Caredet Ltd,Yehuda,Israel), the mean scan time was 23 minutes and 21 seconds and the difference between scan time for the first session and that for the last session was 7 minutes and 26 seconds. Instead with Trios (3Shape, Copenaghen, Danimark), mean scan time was 14 minutes and 25 seconds and the difference between the scan time for the first session and that for the last session was 4 minutes and 23 seconds, considering that the time taken to make a conventional impression was 24 minutes and 42 seconds $(23,24)$. The authors concluded that the time of scanning decreased after repeated scanning with both device and that scanning proficiency increased. However, the experience is a 
difficult parameter to measure. Some manufacturer of intraoral digital scanner suggests 15 to 16 scans to archive the learning curve, but Giménez et al. (15) noted that the inexperienced operators obtained accuracy similar to the experienced ones in their last two impressions which indicates that gaining experience is a very subjective issue. The difference of deviations between the experienced and inexperienced group was $44 \mu \mathrm{m}$. Lee et al. (25) evaluated the difficulty level and operator's perception between dental students and experienced clinicians when making digital and conventional implant impressions. The authors concluded that the conventional impression was more difficult for the student group respect that clinician group, but the difficulty level of digital impressions was same between the groups and the student group favoured the digital impression technique. In our study all the operators were inexperienced because the authors wanted to evaluate the learning curve of digital methodology, but only digital impression technique was performed. A limitation of the present study was that only one digital intraoral scanner system was used for digital impression and only $3 \mathrm{D}$ distance analysis was performed. Further studies should be carried out with different digital intraoral scanners systems to compare the different potential and defects of each.

\section{Conclusion}

The scanning methods have a great influence on the accuracy of digital impressions for abutments in full-arch implant rehabilitation. We decided to use only the scanning method MetB for future scanning with this Ios. The time analysis showed that with the increasing of the number of scans the scan time decreases.

\section{Acknowledgments}

The authors have no commercial or financial dealings that may pose a conflict of interest or potential conflict of interest. The authors thank Ing. MatteoTurchetto for technical support.

\section{References}

1. Christensen GJ. Will digital impressions eliminate the current problems with conventional impressions? J Am Dent Assoc. 2008;139:761-763.

2. Lee H, So JS, Hochstedler JL, Ercoli C. The accuracy of implant impressions: a systematic review. J Prosthet Dent. 2008;100:285-291.

3. Eliasson A, Wennerberg A, Johansson A, Ortorp A, Jemt T. The precision of fit of milled titanium implant frameworks (I-Bridge) in the edentulous jaw. Clin Implant Dent Relat Res. 2010;12:81-90. 
4. Di Fiore A, Meneghello R, Savio G, Sivolella S, Katsoulis J, Stellini E. In Vitro Implant Impression Accuracy Using a New Photopolymerizing SDR Splinting Material. Clin Implant Dent Relat Res. 2015;17(Suppl 2):721-729.

5. Vigolo P, Majzoub Z, Cordioli G. Evaluation of the accuracy of three techniques used for multiple implant abutment impressions. J Prosthet Dent. 2003;89:186-192.

6. Papaspyridakos P, Chen CJ, Gallucci GO, Doukoudakis A, Weber HP, Chronopoulos V. Accuracy of implant impressions for partially and completely edentulous patients: a systematic review. Int J Oral Maxillofac Implants. 2014;29:836-45.

7. Malaguti G, Rossi R, Marziali B, Esposito A, Bruno G, Dariol C, Di Fiore A. In vitro evaluation of prosthodontic impression on natural dentition: a comparison between traditional and digital techniques. Oral Implantol (Rome) 2017;9:21-27.

8. Lee SJ, Gallucci GO. Digital vs. conventional implant impressions: efficiency outcomes. Clin Oral Implants Res. 2013;24:111-115.

9. Di Fiore A, Meneghello R, Graiff L, Savio G, Vigolo P, Monaco C, Stellini E. Full arch digital scanning systems performances for implantsupported fixed dental prostheses: a comparative study of 8 intraoral scanners. J Prosthodont Res 2019;63:396-403.

10. Prasad SM, Maniar HS, Soper NJ, Damiano RJ Jr, Klingensmith ME. The effect of robotic assistance on learning curves for basic laparoscopic skills. Am J Surg 2002;183:702-7

11. Yohannes P, Rotariu P, Pinto P, Smith AD, Lee BR. Comparison of robotic versus laparoscopic skills: is there a difference in the learning curve? Urology 2002;60:39-45.

12. Ender A, Mehl A. Accuracy of complete-arch dental impressions: a new method of measuring trueness and precision. J Prosthet Dent. 2013;109:121-128.

13. Patzelt SB, Emmanouilidi A, Stampf S, Strub JR, Att W. Accuracy of full-arch scans using intraoral scanners. Clin Oral Investig. 2014;18:1687-1694.

14. Güth JF, Keul C, Stimmelmayr M, Beuer F, Edelhoff D. Accuracy of digital models obtained by direct and indirect data capturing. Clin Oral Investig. 2013;17:1201-1208.

15. Giménez B, Özcan M, Martínez-Rus F, Pradíes G. Accuracy of a digital 
impression system based on active wavefront sampling technology for implants considering operator experience, implant angulation, and depth. Clin Implant Dent Relat Res. 2015;17(Suppl 1):54-64.

16. Papaspyridakos P, Gallucci GO, Chen CJ, Hanssen S, Naert I, Vandenberghe B.Digital versus conventional implant impressions for edentulous patients: accuracy outcomes. Clin Oral Implants Res. 2016;27:465-472.

17. Ender A, Mehl A. Influence of scanning strategies on the accuracy of digital intraoral scanning systems. Int J Comput Dent. 2013;16(1):1121.

18. Müller P, Ender A, Joda T, Katsoulis J. Impact of digital intraoral scan strategies on the impression accuracy using the TRIOS Pod scanner. Quintessence Int. 2016;47:343-349

19. Kim J, Park JM, Kim M, Heo SJ, Shin IH, Kim M. Comparison of experience curves between two 3-dimensional intraoral scanners. J Prosthet Dent. 2016 Aug;116(2):221-30.

20. Savio G, Meneghello R, Concheri G. Optical properties of spectable leses computed by surfaces differential quantities. Adv Sci Lett 2013;19:595-600.

21. Chandran DT, Jagger DC, Jagger RG, Barbour ME. Two- and threedimension- al accuracy of dental impression materials: effects of storage time and moisture contamination. Biomed Mater Eng 2010;20:243-249.

22. Ziegler M. Digital impression taking with reproducibly high precision. Int J Comput Dent 2009;12:159-163.

23. Di Fiore A, Vigolo P, Graiff L, Stellini E. Digital vs Conventional Workflow for Screw-Retained Single-Implant Crowns: A Comparison of Key Considerations. Int J Prosthodont. 2018;31:577-579.

24. Kim W, Kim K. Correct methods for using alginate impression materials.

25. Lee SJ, Macarthur RX 4th, Gallucci GO. An evaluation of student and clinician perception of digital and conventional implant impressions. J Prosthet Dent. 2013;110:420-423. 